\title{
LIM homeobox transcription factors, a novel subfamily which plays an important role in cancer (Review)
}

\author{
XI WANG, CHAO HE and XIAOTONG HU \\ Biomedical Research Center, Sir Run Run Shaw Hospital, Zhejiang University and Key Laboratory \\ of Biotherapy of Zhejiang Province, Hangzhou, Zhejiang 310016, P.R. China \\ Received January 12, 2014; Accepted March 13, 2014
}

DOI: $10.3892 /$ or.2014.3112

\begin{abstract}
LIM homeobox genes are one of the most important subfamilies of homeobox genes which encode LIM-homeodomain (LIM-HD) proteins featuring two LIM domains in their amino termini and a centrally located HD that is used to interact with specific DNA elements in target genes. Numerous studies have reported their fundamental roles in the development of various organisms; however, little is known about their functions in cancer. Recently, research has shown that LIM homeobox genes also play an important role in cancer development. Among 12 human LIM homeobox genes, 10 LIM-HD proteins have been reported to be associated with cancer. In the present review, we mainly summarize the functions of these genes in various types of cancer and their potential as biomarkers and the related challenges. More in-depth research concerning LIM homeobox genes in cancer from a signaling pathway perspective may help to understand tumor profiles, establish biomarkers and guide choices for combinatorial drug therapies.
\end{abstract}

\section{Contents}

1. Introduction

2. Relationship of LIM homeobox genes and cancer

3. Targeting the developmental pathways to explore the role of LIM homeobox genes in cancer

4. The potential of LIM homeobox genes as cancer biomarkers and the challenges

5. Conclusions

Correspondence to: $\mathrm{Dr}$ Xiaotong Hu, Biomedical Research Center, Sir Run Run Shaw Hospital, Zhejiang University and Key Laboratory of Biotherapy of Zhejiang Province, 3 Qingchun East Road, Hangzhou, Zhejiang 310016, P.R. China

E-mail: hxt_hangzhou@sina.com

Key words: LIM homeobox genes, biomarkers, signaling pathway, cancer

\section{Introduction}

LIM homeobox genes are one of the most important subfamilies of homeobox genes. They encode a series of LIM-homeodomain (LIM-HD) proteins featuring two LIM domains in their amino termini and a centrally located HD that is used to interact with specific DNA elements in target genes (Fig. 1). This special structure can set LIM-HD proteins apart from other transcription factors of homeodomain superfamilies such as paired homeobox (PAX) proteins, Pit-Oct-Unc (POU)-HD and HOX (homeobox) cluster proteins $(1,2)$. Their LIM domain contains two tandemly-repeated, cysteine-rich, double-zinc finger motifs that can be recognized by a number of co-factors which can mediate LIM-HD functions. These LIM domain-interacting proteins include NLI/LDB/CLIM/ CHIP, MRG1, SLB and RLIM, a ubiquitin protein ligase. What is more, by means of the LIM domain, LIM-HD proteins can interact with other transcription regulators; thus, they can participate in a wide array of developmental events (3-6).

Mammalian genomes such as those of the mouse, rat and human contain at least 12 LIM homeobox genes (Table I) that encode key regulators of developmental pathways. Studies with mouse models and human patients have shown that these genes are critical for the development of specialized cells in multiple tissue types including the nervous system, skeletal muscle, the heart, the kidneys and endocrine organs such as the pituitary gland and the pancreas (1).

Although many researchers have reported their fundamental roles in the development of various organisms, little is known concerning the functions of these LIM-HD proteins in cancer. Recently, studies have shown that LIM homeobox genes also play an important role in cancer. Among 12 human LIM homeobox genes, 10 LIM-HD proteins have been reported to be associated with various types of cancer (Table II). In this review, we mainly summarize the functions of human LIM-HD proteins in cancer development and their potential as biomarkers and the related challenges. In addition, we discuss the role of these LIM homeobox genes in cancer from a signaling pathway perspective.

\section{Relationship of LIM homeobox genes and cancer}

LHX1 and cancer. LHX1, also called LIM1, is required for head, kidney and female reproductive tract development in 
Table I. Human LIM homeobox genes in the HUGO Gene Nomenclature Committee (HGNC) database.

\begin{tabular}{|c|c|c|c|}
\hline HGNC name & Symbol & Synonym symbol(s) & Chromosomal location \\
\hline LIM homeobox 1 & LHX1 & $\begin{array}{l}\text { LIMK4, LIM-1, LIM1, } \\
\text { MGC126723, MGC138141 }\end{array}$ & $17 q 12$ \\
\hline LIM homeobox 2 & LHX2 & LH2, MGC138390 & $9 q 33.3$ \\
\hline LIM homeobox 3 & LHX3 & $\begin{array}{l}\text { DKFZp762A2013, LIM3, } \\
\text { M2-LHX3, CPHD3 }\end{array}$ & $9 \mathrm{q} 34.3$ \\
\hline LIM homeobox 4 & LHX4 & Gsh-4, Gsh4 & $1 \mathrm{q} 25.2$ \\
\hline LIM homeobox protein 5 & LHX5 & MGC129689 & $12 \mathrm{q} 24.31-\mathrm{q} 24.32$ \\
\hline LIM homeobox protein 6 & LHX6 & $\begin{array}{l}\text { LHX6.1, MGC119542, } \\
\text { MGC119544, MGC119545 }\end{array}$ & $9 \mathrm{q} 33.2$ \\
\hline LIM homeobox 8 & LHX8 & Lhx7 & $1 \mathrm{p} 31.1$ \\
\hline LIM homeobox 9 & LHX9 & / & $1 \mathrm{q} 31.3$ \\
\hline ISL LIM homeobox 1 & ISL1 & ISL-1, ISLET1 & $5 q 11.1$ \\
\hline ISL LIM homeobox 2 & ISL2 & FLJ10160, ISL-2 & $15 \mathrm{q} 24.3$ \\
\hline LIM homeobox transcription factor $1, \alpha$ & LMX1A & LMX1-1, LMX-1, LMX1 & $1 q 22-q 23$ \\
\hline LIM homeobox transcription factor $1, \beta$ & LMX1B & $\begin{array}{l}\text { LMX1-2, NPS1, } \\
\text { MGC142051, MGC138325 }\end{array}$ & $9 q 33.3$ \\
\hline
\end{tabular}

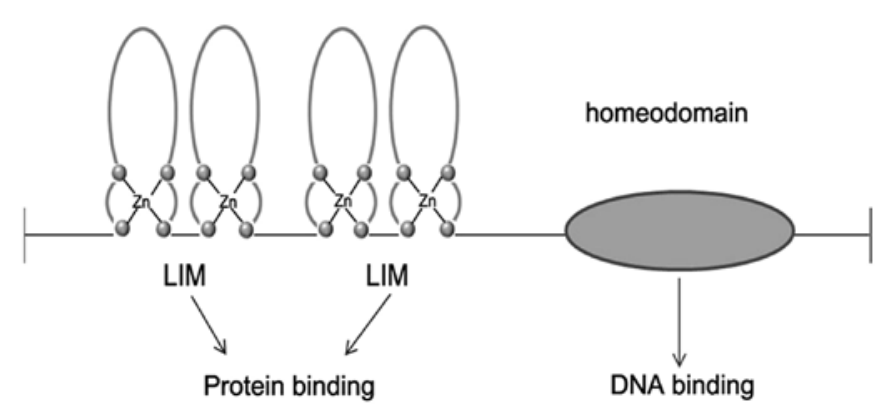

Figure 1. Schematic structure of LIM-HD proteins. The small gray spheres indicate the zinc-binding residues.

the murine embryo (7-9). Most LIM1 null mutants die with a headless phenotype around E10.5; the few surviving newborns had no kidney (7). Conditional ablation of LIM1 in the metanephric mesenchyme blocks the formation of nephrons at the nephric vesicle stage, leading to the production of small, nonfunctional kidneys that lack nephrons (10).

Guertl et al (11) investigated LIM1 expression during human renal development, in dysplastic kidneys and in renal neoplasms and found that LIM1 was detected in pretubular aggregates, S-shaped and comma-shaped bodies as well as immature glomeruli between 10 and 30 weeks of gestation. Eleven dysplastic kidneys showed no expression of LIM1. In contrast, 12 of 32 nephroblastomas showed nuclear positivity. One regressive nephroblastoma had diffuse expression of LIM1 in tubular structures, all others showed focal positivity in mesenchymal, blastemal and epithelial structures. Renal cell carcinomas revealed no expression of LIM1. Their study supports the concept of a causative role of LIM1 deficiency in the development of multicystic kidney. In a small subset of nephroblastomas with a more diffuse expression pattern, LIM1 might also contribute to the pathogenesis of these lesions.
Dormoy et al (12) further assessed whether LIM1 may be associated with tumorigenesis. They found that LIM1 is constitutively and exclusively reexpressed in tumors as a growth and survival factor in human clear cell renal cell carcinoma (CCC). More importantly, in nude mice bearing human CCC, LIM1 silencing abolished tumor growth through the same mechanism as in vitro. In LIM1-depleted cells and tumors, cell motility was substantially impaired due to the inhibition of expression of various proteins involved in metastatic spread, such as paxillin or tenascin-C.

LHX2 and cancer. The LHX2 gene functions as a transcriptional regulatory protein in the control of lymphoid and neural cell differentiation. LHX2 was initially identified as an early marker in B-lymphocyte differentiation (13). Overexpression of LHX2 in murine hematopoietic precursors leads to the development of chronic myeloproliferative disorders (14).

LHX2 was once found to be deregulated by juxtaposition with the IGH locus in a pediatric case of chronic myeloid leukemia (CML) in B-cell lymphoid blast crisis. The strong overexpression of LHX2 induced by $\mathrm{t}(9 ; 14)(\mathrm{q} 33 ; \mathrm{q} 32)$ may play a recurrent role in leukemogenesis, specifically when it is in association with BCR-ABL chimera of the Philadelphia chromosome ( $\left.\mathrm{Ph}^{\prime}\right)$, the hallmark of CML (15).

High levels of LHX2 expression were also observed in all cases of CML tested, regardless of disease status and may prove useful as a marker of CML for monitoring residual disease (16). Since LHX2 was mapped to chromosome 9q33-34.1, in the same region as the reciprocal translocation that creates the BCR-ABL. they also confirmed that the transcriptional activation of LHX2 in CML is likely due to a cis-acting effect, but not a trans-acting effect, of the Bcr-Abl fusion protein. The high level of LHX2 mRNA in CML cells probably can also be a consequence of the low level of methylation of the gene in leukemic cells (17). 
Gorantla et al (18) reported the presence of uPA in the nucleus as well as the interaction of uPA with LHX2. LHX2 was overexpressed at the invasive front of tumors while completely absent in tumors downregulated for both uPAR and uPA. Suppression of the UPAR-uPA system retards angiogenesis, invasion, and in vivo tumor development in pancreatic cancer cells. Recently, LHX2 was also identified as a transcription factor functionally positioned downstream of $\mathrm{p} 63$ and $\mathrm{NF}-\kappa \mathrm{B}$, but upstream of signals such as Wnt/ $\beta$-catenin, $\mathrm{BMP}$, and $\mathrm{SHH}$ that are required to drive activated stem cells toward terminal differentiation (19). This would indicate that LHX2 may behave like a gatekeeper molecule mediating the activation of cancer stem cells.

Notably, besides being overexpressed as an oncogene in cancer, LHX2 may be downregulated due to hypermethylation in cancer. Homeobox genes that are upregulated in cancer may be normally expressed during development and/or in undifferentiated cells, whereas others that are downregulated in cancer may be normally expressed in adulthood and/or in differentiated tissues (20).

Rahmatpanah et al (21) used an array-based technique called differential methylation hybridization (DMH) to study small B-cell lymphoma (SBCL) subtypes and found that hypermethylation of only one gene, LHX2, was present in all Non-Hodgkin's lymphoma (NHL) cell lines and a high proportion of patient samples.

Kim et al (22) utilized a genome-wide technique, methylated DNA isolation assay (MeDIA), in combination with high-resolution $\mathrm{CpG}$ microarray analysis to identify hypermethylated genes in breast cancer. LHX2 showed significantly higher frequencies of aberrant hypermethylation in primary tumors $(43.6 \%$; $\mathrm{P}<0.05)$ while frequencies were intermediate in paired adjacent normal tissues and absent in normal tissues. Moreover, a significant number of genes $(2853$; $\mathrm{P}<0.05)$ exhibited an expression-methylation correlation in breast cancer. Among these genes, LHX2 and LHX5 had prognostic value independent of subtypes and other clinical factors (23).

In lung cancer, Rauch et al (24) identified frequent methylation of homeodomain-containing genes including LHX2 and LHX4 using methylated-CpG island recovery assay (MIRA)-assisted microarray analysis. Although low levels of methylation were detected in some normal tissues removed by tumor surgery, methylation of LHX2 and LHX4 was more pronounced in tumor samples.

LHX3 and cancer. LHX3 null mice were found to have defective development of spinal cord motor neurons (25) and also to exhibit incomplete pituitary development $(26,27)$. LHX3 functions to specify interneuron and motor neuron fates during development (28). To date, LHX3 has only been shown to be correlated with breast cancer. Dietrich et al (29) reported that elevated methylation of LHX3 was detected in invasive ductal carcinoma (IDC) and ductal carcinoma in situ (DCIS) compared with normal ducts, adenosis tissue, stroma and tumor infiltrating lymphocytes (TILs).

LHX4 and cancer. During embryonic and postnatal development, the LHX4 gene is expressed in hindbrain, cerebral cortex, pituitary gland and spinal cord, suggesting that LHX4 plays a role in nervous system development $(30,31)$. Mutations in LHX4 genes are associated with combined hormone deficiency diseases in human and animal models $(32,33)$.

Similar to LHX2, LHX4 can be upregulated by chromosomal translocations and downregulated by hypermethylation in cancer. Firstly, LHX4 mRNA was found to be expressed at high levels in the leukemic cells of patients and in an acute lymphoblastic leukemia (ALL) cell line due to the $t(1 ; 14)$ (q25; 32 ) suggesting a rare but recurrent genetic abnormality in leukemogenesis $(34,35)$.

Moreover, de Bruijn et al (36) reported that the SS18 gene on chromosome 18 is fused to either one of the three closely related SSX genes on the $\mathrm{X}$ chromosome as a result of the synovial sarcoma-associated $\mathrm{t}(\mathrm{X} ; 18)$ translocation. They found that endogenous LHX4 binds to the CGA promoter and that LHX4-mediated CGA activation is enhanced by the SS18-SSX protein, but not by the SSX protein and suggested that this novel protein-protein interaction may have direct consequences for the deregulation of SS18-SSX target gene LHX4 in the development of human synovial sarcomas.

Meanwhile, Goc et al (37) provided an initial report that simvastatin simultaneously modulated intrinsic and extrinsic pathways in the regulation of prostate cancer cell apoptosis in vitro and in vivo and also significantly reduced protein levels of pro-survival gene LHX4, but the exact function is yet to be determined.

In contrast, low expression of LHX4 was found in primary lung tumor samples (23) and hepatocellular carcinoma (HCC) (38) when compared with the expression level in paired non-cancerous tissues. Moreover, low expression of LHX4 was associated with tumor undifferentiation state and a high AFP level in HCC. Functional studies revealed that ectopic expression of LHX4 reduced AFP expression, which leads to the suppression of HCC growth. The results indicate that LHX4 functions as a potential tumor suppressor in hepatocarcinogenesis.

LHX5 and cancer. Human LHX5 is expressed in the adult central nervous system, including the spinal cord, thalamus and the cerebellum (39). The mouse LHX5 protein is closely related to the LHX1 LIM-HD factor, and complementary or overlapping roles of these two regulatory proteins have been suggested (40). Mice that are heterozygous for a LHX5 gene disruption appear normal, while most homozygous mice die a few days after birth (41).

Together with LHX2, LHX5 was found to have prognostic value independent of subtypes and other clinical factors in breast cancer (22).

LHX6 and cancer. LHX6 encodes an LIM-homeodomain protein involved in embryogenesis, more specifically in mammalian head development (42). Estécio et al (43) identified LHX6 as a new frequent cancer-associated hypermethylated $\mathrm{CpG}$ island in human head and neck squamous cell carcinoma (HNSCC). The hypermethylation of this fragment was detected in 13 of $14(92.8 \%)$ HNSCC cell lines studied and in 21 of 32 $(65.6 \%)$ primary tumors, whereas little or no methylation was noted in 10 normal oral mucosa samples. They extended this investigation to other cancer cell lines and methylation was found in those derived from colon, breast, leukaemia, lung and in $12 / 14$ primary colon tumors. 


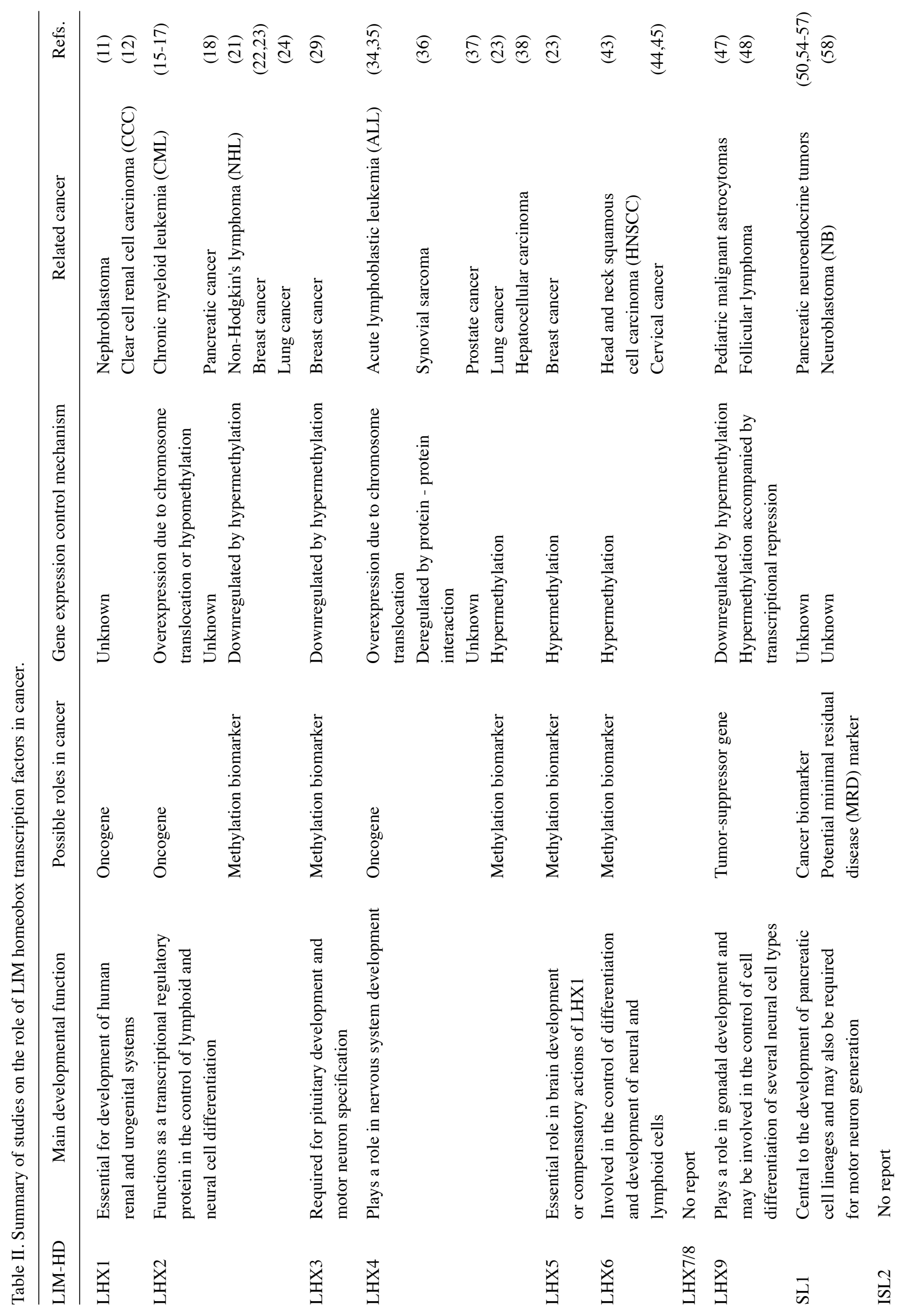


LHX6 methylation was also found in cervical cancer cell lines and cancer tissues (44). This epigenetic alteration in the LHX6 promoter begins at a relatively early stage as CIN I, suggesting its potential as a biomarker for early diagnosis (45). Moreover, overexpression of the LHX6 gene in cervical cancer cells was found to suppress the tumorigenic phenotype, as shown by soft agar colony formation and migration assays, suggesting that LHX6 could be a novel tumor-suppressor gene in the cervix.

LHX9 and cancer. The expression pattern and structural characteristics of LHX9 suggest that it encodes a transcription factor that might be involved in the control of cell differentiation of several neural cell types (46). The LHX9 gene is frequently silenced in pediatric malignant astrocytomas by hypermethylation and this epigenetic alteration is involved in glioma cell migration and invasiveness (47). LHX9 hypermethylation accompanied by transcriptional repression was found in follicular lymphoma (48).

ISL1 and cancer. The ISL1 transcription factor was initially cloned from pancreatic insulin-producing cells where it is able to bind the insulin gene enhancer (49). Mice deficient in ISL1 fail to form the dorsal exocrine pancreas, and islet cells fail to differentiate (50). In addition to its roles in the pancreas and heart, ISL1 is one of the earliest markers for motor neuron differentiation (51).

Thus, it is not surprising that ISL1 has been shown to be a sensitive marker of pancreatic islet cells and their neoplasms (50,52-56). ISL1 has been reported as a sensitive lineagespecific marker for pancreatic neuroendocrine neoplasms (NENs) and their metastases. Graham et al (54) also studied its specificity with large numbers of NENs from other parts of the gut or other organs. They found that ISL1 does not distinguish pancreatic NENs from duodenal and colorectal NENs, even when used in association with CDX2. On the other hand, in order to better understand the expression of the four transcription factors (TFs): ISL1, pancreatico-duodenal homeobox 1 gene product (PDX1), neurogenin 3 gene product (NGN3), and CDX-2 homeobox gene product (CDX2), that mainly govern the development and differentiation of the pancreas and duodenum, Hermann et al (57) studied their expression in hormonally defined pancreatic neuroendocrine tumors (P-NETs) and duodenal neuroendocrine tumors (D-NETs). They found a correlation between TF expression patterns and certain hormonally defined P-NET and D-NET types, suggesting that most of the tumor types originate from embryologically determined precursor cells. However, the observed TF signatures cannot distinguish P-NETs from D-NETs.

In addition, Cheung et al (58) explored potential minimal residual disease (MRD) markers differentially expressed in neuroblastoma (NB) tumors over normal marrow/blood with genome-wide expression profiling and identified 8 top-ranking markers: CCND1, CRMP1, DDC, GABRB3, ISL1, KIF1A, PHOX2B and TACC2. They were abundantly expressed in stage 4 NB tumors $(n=20)$ and had low to no detection in normal marrow/blood samples $(n=20)$. Moreover, expression of CCND1, DDC, GABRB3, ISL1, KIF1A and PHOX2B in 116 marrows sampled after 2 treatment cycles 
was highly prognostic of progression-free and overall survival $(\mathrm{P}<0.001)$.

LMX1A and cancer. The LMX1A gene maps to 1q22-q23 and was proven to be a critical regulator of cell-fate decisions using genetic fate mapping in wild-type and LMX1A ${ }^{--}$mice (59). LMX1A was also found to play a pivotal role in the differentiation of human embryonic stem cells into midbrain dopaminergic neurons (60).

Recent evidence has shown an important role of LMX1A in cancer. For example, Lin et al (61) reported that higher immunostaining scores and the percentage of cells stained for LMX1A in mucinous cystadenocarcinomas correlated with T stage, American Joint Committee on Cancer clinical stage, poorer tumor differentiation, and poorer survival rate. In addition, a higher intensity of immunoreactivity for LMX1A correlated with more advanced grade in WHO grade I-III gliomas, but not in WHO grade IV tumors (62). In addition, higher expression of LMX1A and OPN was found to be highly correlated with histologic grade and pathologic stage of pancreatic ductal adenocarcinomas (63).

In contrast, LMX1A was identified as a metastasis suppressor in cervical cancer (64-66). It was once reported that the methylation of LMX1A correlated with the recurrence and overall survival due to the mechanisms affecting epithelialmesenchymal transition (EMT) and stem-like properties in ovarian cancer $(67,68)$. The methylation of LMX1A was also found to be associated with bladder cancer recurrence (69). Dong et al (70) found that the expression of LMX1A was significantly decreased due to the hypermethylation in gastric cancer tissues compared with normal tissues. Restoration of LMX1A induced cell apoptosis and suppressed anchorageindependent growth, suggesting that LMX1A is a potential biomarker for gastric cancer. Moreover, LMX1A hypermethylation was reported in a colon cancer cell line (HCT-116), but was demethylated in DKO cells in which two major DNA methyltransferases, DNMT1 and DNMT3b, were genetically disrupted (71). However, its role in transformation has not yet been characterized.

LMX1B and cancer. Together with LMX1A, LMX1B is part of a related subfamily of LIM-HD genes. Using targeted disruption of the mouse LMX1B gene, multiple functions of this factor have been uncovered. Mice lacking functional LMX1B exhibit numerous abnormalities, including a lack of ciliary body, iris stroma and corneal dysplasia $(72,73)$.

To date, LMX1B has only been suggested to be associated with breast cancer. Rieger et al (74) found that LBH was overexpressed in highly invasive ER-negative, basal subtype human breast cancers and suppressed the differentiation of HC11 mammary epithelial cells. In further support of a clinical association of LBH with Wnt/ $\beta$-catenin signaling, additional meta-analysis showed that LBH overexpression also correlates with Wnt pathway gene expression in colon cancer, which is primarily driven by Wnt activating mutations. Wnt7aLMX1B signaling may be an important repressive mechanism that blocks Wnt/ $\beta$-catenin target gene expression, and consequently ventral differentiation, in dorsal limb ectoderm (75). Thus, LBH may act as a downstream effector of this signaling in both normal and neoplastic epithelial development, which is under the tight control of antagonistic non-canonical Wnt7a signaling (76).

\section{Targeting the developmental pathways to explore the role of LIM homeobox genes in cancer}

Although numerous LIM homeobox genes are known to be involved in cancer, the roles of these genes in cancer remain unclear. Few LIM homeobox gene signaling pathway studies in cancer are available. Among these, LMX1A and LIM1 have been the subjects of a certain degree of intensive study.

Lai et al (65) demonstrated that LMX1A may have a critical role in preventing cervical cancer invasion and metastasis by inhibiting different aspects of EMT. They found that besides inhibiting cervical cancer invasion, the restoration of LMX1A altered expression of epithelial and mesenchymal markers in two cervical cancer cell lines (HeLa-3rd and CaSki). Furthermore, by analyzing TGF- $\beta$ signaling, they found that BMP4 and BMP6 were downregulated by LMX1A. BMPs have been confirmed to be important components of LMX1A-dependent roof plate signaling, in which BMP6 is dependent on LMX1A expression during spinal cord development in mice (77). Moreover, LMX1A was found to be a mediator of early BMP signaling, and its activation of early roof plate development is dependent on BMP4 signaling in chicks (78). These complex regulatory networks of different BMPs and LMX1A in diverse microenvironments and tissues during embryonic development may also hint to its roles in cancer biology.

Transcription factors such as SNAIL, SLUG, TWIST, ZEB and FOXC2 are important markers of EMT. There is much evidence to confirm that several oncogenic pathways, such as growth factors (GFs), Ras, SHH, TGF- $\beta$, Wnt and Notch, may induce EMT. LIM homeobox genes such as LMX1A may also be important intermediate transcription factors which can induce or regulate core transcription factors and co-operate with them in target regulation, eventually leading to downregulation of epithelial genes and upregulation of mesenchymal genes (Fig. 2).

Regarding LIM1, it was found to be a downstream effector of SHH-Gli signaling functions and its expression is regulated by Pax2, FGFs and Wnt factors, which were confirmed to be involved in human tumorigenesis (79). Recently, LIM1 was found to be a growth and survival factor in human clear cell renal cell carcinoma through the activation of multiple oncogenic pathways including phosphoinositide kinase-3/Akt, MAPK and NK- $\mathrm{KB}$ pathways (13). To elucidate additional mechanisms accounting for the effect of LIM1 on tumor cell growth, western blot analysis and Proteome Profiler arrays (coated with apoptotic, phosphoproteins or angiogenesis pathways/markers) were used. The results showed that the various signaling pathways and molecular factors involved in cell apoptosis, proliferation, movement, angiogenesis were regulated at the level of expression and/or activation directly or indirectly by LIM1, such as phosphorylated Akt, Stats, cytochrome $c$, Fas, p38, p53, FGF, PDGF and EGF receptors as well as markers of cell movement, MMP8 andMMP9. Taken together, we suggest that LIM1 has a vital role in the expression and/or activation of various oncogenic and angiogenic pathways in human CCC (Fig. 3). 


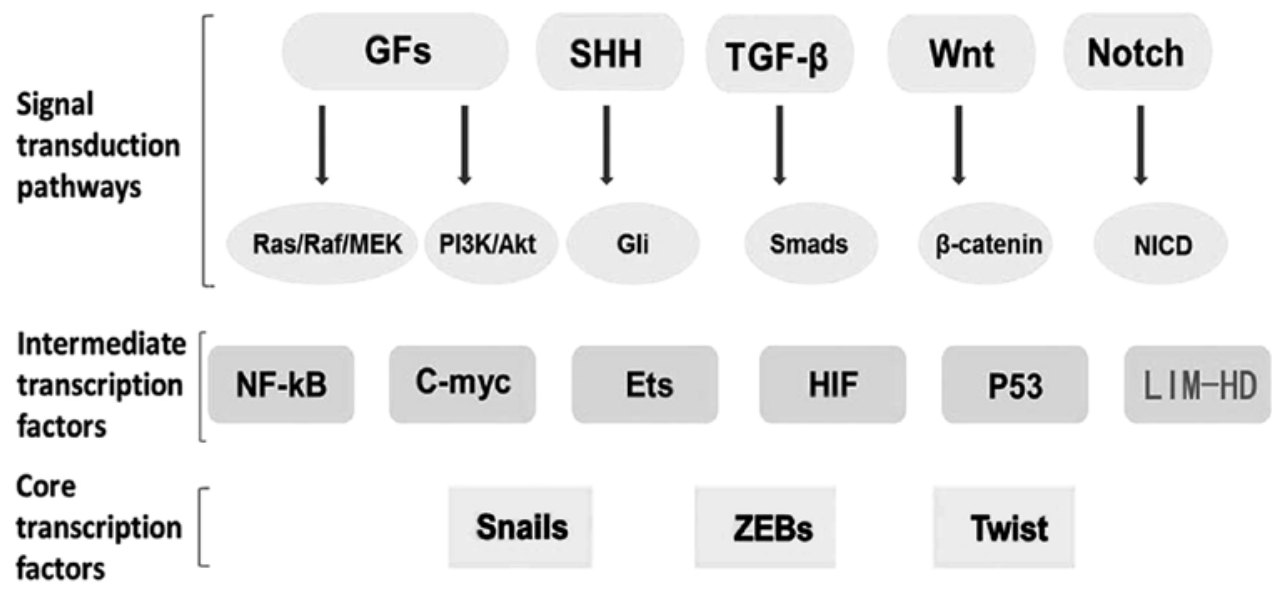

Figure 2. A conceptual layer overview of cancer EMT. LIM homeobox genes such as LMX1A may also be important intermediate transcription factors which can induce or regulate core transcription factors and co-operate with them in target regulation, eventually leading to downregulation of epithelial genes and upregulation of mesenchymal genes.

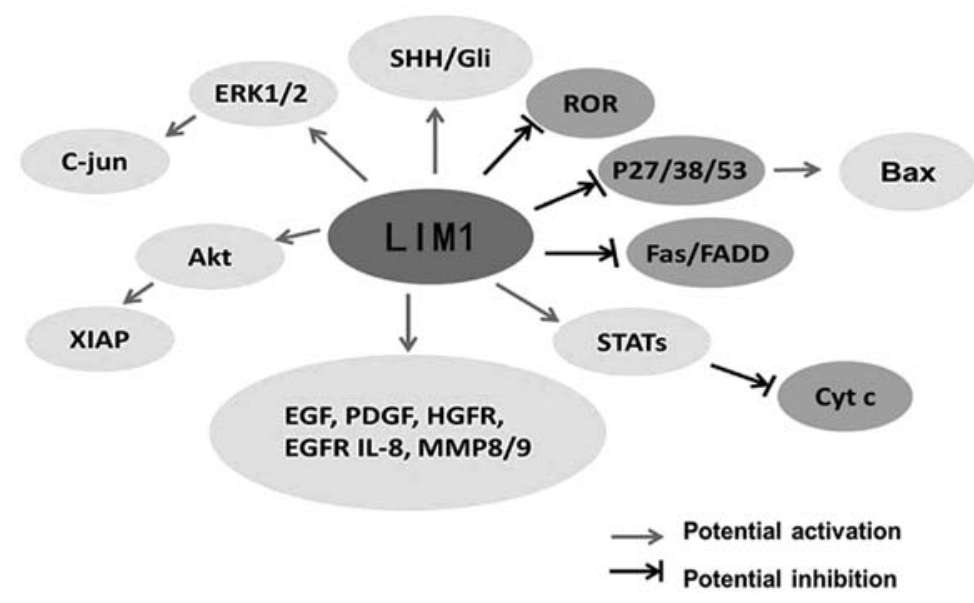

Figure 3. Potential LIM1 targets identified by Proteome Profiler array and western blot analysis in human clear cell renal cell carcinoma.

Since the developmental pathways involved in the genesis and the growth of an organ are also responsible for the development of a tumor, the idea that tumors 'hijack' for their own growth signaling pathways involved in normal development is emerging (80). The few available LIM homeobox gene signaling pathway studies in cancer reviewed above are consisted with this concept. Cancer often arises when normal cellular growth goes awry due to defects in critical signal transduction pathways; thus targeting these developemental pathways to explore the role of LIM homeobox genes in cancer will be effective.

In the present study, we document various confirmed signal pathway studies in development involving LIM homeobox transcription factors, which could provide various clues to explore the role of LIM homeobox genes in cancer.

Sato et al (81) reported that the kinase activity of WNKs is required for induction of LHX8 gene expression and the activation of SPAK/OSR1, and that the kinase-dead form of WNK acts as an actual dominant-negative form in the signaling pathway. Furthermore, the expression of LHX8 by either hypertonic or RA stimulation was required for the expression of both WNK1 and WNK4. Thus, this study provides initial evidence identifying the LHX8 gene acting downstream in the
WNK-SPAK/OSR1 pathway, and demonstrates the significance of the WNK-OSR1-LHX8 pathway in neural development. WNK is a family of serine/threonine protein kinases that are characterized by a typical sequence variation within the conserved catalytic domain and could phosphorylate and activate SPAK or OSR1 kinases $(82,83)$. The WNK-SPAK/OSR1 pathway is known to regulate various ion co-transporters and is widely conserved among many species $(84,85)$. WNK1 is also required for cell division in cultured cells (86), and proliferation, migration and differentiation of neural progenitor cells (87). There is growing evidence for additional roles of WNK kinases in various signaling cascades related to cancer (88).

Wnt gain of function in cardiac progenitor cells was found to lead to expansion of ISL1-positive progenitors with a concomitant increase in FGF signaling through activation of a specific set of FGF ligands including FGF3, FGF10, FGF16 and FGF20. These data reveal that $\mathrm{Wnt} / \beta$-catenin signaling promotes expansion of ISL1-positive progenitor cells through regulation of FGF signaling (89).

Roof plate (RP) development has been well studied in the spinal cord, where its specification relies on interactions between inductive signals of the TGF- $\beta$ family produced by the adjacent epidermal ectoderm and intrinsic homeodomain 
Table III. The methylation rate of LIM homeobox transcription factors in cancer cell lines and/or tumor tissues.

\begin{tabular}{|c|c|c|c|}
\hline LIM-HD & Related cancer & Methylation rate & Refs. \\
\hline \multirow[t]{3}{*}{ LHX2 } & NHL & B-CLL/SLL, 46.6\%; MCL, 41.6\%; FL,73\% & $(21)$ \\
\hline & Breast cancer & Tumor biopsies, $43.6 \%$ & $(22,23)$ \\
\hline & Lung tumor & Tumor biopsies, $58 \%$ & $(24)$ \\
\hline LHX3 & Breast cancer & Unknown & $(29)$ \\
\hline \multirow[t]{2}{*}{ LHX4 } & Lung tumor & Tumor biopsies, $75 \%$ & $(23)$ \\
\hline & Hepatocellular carcinoma & Unknown & $(38)$ \\
\hline LHX5 & Breast cancer & Unknown & $(23)$ \\
\hline \multirow[t]{3}{*}{ LHX6 } & HNSCC & Cell lines, $92.8 \%$; tumor biopsies, $65.6 \%$ & $(43)$ \\
\hline & Colon cancer & Tumor biopsies, $85.7 \%$ & $(43)$ \\
\hline & Cervical cancer & Cell lines, $87.5 \%$ & $(44,45)$ \\
\hline \multirow[t]{3}{*}{ LHX9 } & Pediatric malignant astrocytomas & High-grade gliomas, $55.6 \%$; & $(47)$ \\
\hline & & low-grade gliomas, $29 \%$ & $(48)$ \\
\hline & Follicular lymphoma & Unknown & \\
\hline \multirow[t]{5}{*}{ LMX1A } & Cervical cancer & Tumor biopsies, $89.9 \%$; cervical scrapings, $36 \%$ & $(64-66)$ \\
\hline & Ovarian cancer & Benign, $1.3 \%$; borderline, $7.1 \%$; malignancy, $34.9 \%$ & $(67,68)$ \\
\hline & Bladder cancer & Tumor biopsies, $9.43 \%$ & $(69)$ \\
\hline & Gastric cancer & Tumor biopsies, $82 \%$ & $(70)$ \\
\hline & Colon cancer & Carcinomas, $55 \%$; adenomas, $42 \%$; cell lines, $75 \%$ & $(71)$ \\
\hline
\end{tabular}

NHL, non-Hodgkin's lymphoma; B-CLL, B-cell chronic lymphocytic leukemia; SLL, small lymphocytic lymphoma; MCL, mantle cell lymphoma; FL, follicular lymphoma; HNSCC, human neck squamous cell carcinoma.

transcription factors LMX1A and LMX1B (90-92), while similar mechanisms are involved in RP differentiation in the anterior midbrain. Alexandre et al (93) reported that the plasticity of the midbrain RP derives from two apparently antagonistic influences of FGF8. On the one hand, FGF8 widens beyond the neural folds the competence of the neuroepithelium to develop a RP by inducing the expression of LMX1B and Wnt1. On the other hand, FGF8 exerts a major destabilizing influence on RP maturation by controlling signaling by members of the TGF- $\beta$ superfamily belonging to the BMP, GDF and activin subgroups.

A hallmark of cancer is reactivation/alteration of pathways that control cellular differentiation during developmental processes. Aberrant activation of developmental pathways such as Wnt, Hedgehog and Notch contributes to cancer development and progression, and these pathways are even intertwined at the molecular level. Thus, valuable prognostic biomarkers and the innovative therapies for cancer may be identified through these studies.

\section{The potential of LIM homeobox genes as cancer biomarkers and the challenges}

It is known that the genes that play important roles in the various steps of development may later be overexpressed or downregulated contributing to carcinogenesis. As reviewed above, several groups have reported the use of LIM homeobox genes as diagnostic and prognostic biomarkers, as certain gene expression profiles can be linked to tissue specificity, associated with early stages of carcinogenesis, and even linked to therapy-resistant disease resulting in a worse prognosis.
Analysis of the LIM homeobox gene abnormalities in cancer has demonstrated the presence of overexpression, at times, due to chromosomal translocations. However, more LIM homeobox genes are frequently downregulated in cancer due to hypermethylation (Table III), but not deletion or mutation.

Aberrant hypermethylation of the promoter regions of specific genes is a key event in the formation and progression of cancer and can be applied to cancer diagnostics in three ways: as a marker to detect cancer cells or cancer-derived DNA; as a marker to predict prognosis; and as a biomarker for the assessment of therapeutic response. Methylation analysis has an advantage in that it can be performed using chemically stable DNA (compared to RNA). Moreover, detecting gene methylation is easier than detecting gene mutation since the exact location of a mutation is usually unknown, making it difficult to specifically amplify DNA molecules with an embedded mutation in excess of wild-type molecules. More interestingly, detection of aberrant methylation can provide confirmation of the presence of intact cancer cells or cancer-derived DNA in bodily fluids, such as blood, urine, sputum, saliva and stool. Thus, their potential as biomarkers is growing.

Although numerous studies have demonstrated that LIM homeobox genes may serve as cancer biomarkers for diagnosis and prognosis, there are still many factors limiting the clinical application of these biomarkers. First, the majority of these studies were almost low level clinical reports, and lack of large sample, multi-center clinical studies exists. In order to confirm their clinical utility, enlarged sample size, more prospective studies, detection of the expression of methylated LIM homeobox genes in various periods of tumorigenesis and 
testing using an independent set of collected patient samples must be carried out. Second, the mechanisms involving LIM homeobox genes and cancer are not totally understood, thus more in-depth study is required. Third, the technology analyzing gene methylation is not mature enough. Quantitative analysis of tumor sample is still a challenge. In the future, improving research methods and techniques is necessary. Fourth, as biomarkers, further detailed investigation is required to determine which markers have high sensitivity and specificity. Moreover, because of tumor heterogeneity, no single marker will likely be adequate. The inclusion of more new markers in a panel of hypermethylated genes in cancer potentially increases the sensitivity and specificity of tumor detection. Finally LIM homeobox gene immunohistochemical assessment or methylation detection should be combined with clinical and radiologic information to arrive at a definitive diagnosis.

\section{Conclusions}

LIM homeobox genes are one of the most important subfamilies of homeobox genes. As reviewed above, they not only participate in a wide array of developmental events, but also act as tumor-suppressor genes or oncogenes and are involved in various signaling pathways. Moreover, the silencing of LIM homeobox genes caused by hypermethylation is highly correlated with cancer. Yet, the mechanisms involved in the inhibition or promotion of tumorigenesis by LIM homeobox genes require further in-depth research. It has been reported that LIM homeobox genes may be used as cancer biomarkers for early diagnosis and prognostic evaluation. However, due to the complexity of clinical tumor samples, sensitivity and specificity of LIM homeobox genes have become the main challenges which hinder their clinical application. We believe that more in-depth research concerning LIM-homeobox genes in cancer from a signaling network perspective will be highly valuable in helping to understand tumor profiles, establish biomarkers, and guide choices for combinatorial drug therapies.

\section{Acknowledgements}

The present study was supported by the National Natural Science Foundation of China (grant nos. 81071651 and 81372622), the Zhejiang Provincial Natural Science Foundation of China (grant no. R2100213), the Program for Zhejiang Leading Team of S\&T Innovation (grant no. 2012R10046-03), the Major State Basic Research Development Program (grant no. 2010CB834303), the National High Technology Research and Development Program of China (grant no. 2012AA02A601), the Major Projects in Zhejiang Province (grant no. 2012C13014-1), and the Fundamental Research Funds for the Central Universities (grant no. 2012FZA7020).

\section{References}

1. Hobert $\mathrm{O}$ and Westphal H: Functions of LIM-homeobox genes. Trends Genet 16: 75-83, 2000.

2. Dawid IB: LIM domain proteins. C R Acad Sci 3: 295-306, 1995.

3. Bach I: The LIM domain: regulation by association. Mech Dev 91: 5-17, 2000.
4. Glenn DJ and Maurer RA: MRG1 binds to the LIM domain of Lhx2 and may function as a coactivator to stimulate glycoprotein hormone alpha-subunit gene expression. J Biol Chem 274: 36159-36167, 1999 .

5. Howard PW and Maurer RA: Identification of a conserved protein that interacts with specific LIM homeodomain transcription factors. J Biol Chem 275: 13336-13342, 2000.

6. Ostendorff HP, Peirano RI, Peters MA, et al: Ubiquitinationdependent cofactor exchange on LIM homeodomain transcription factors. Nature 416: 99-103, 2002.

7. Shawlot $\mathrm{W}$ and Behringer RR: Requirement for Lim1 in headorganizer function. Nature 374: 425-430, 1995.

8. Karavanov AA, Karavanova I, Perantoni A and Dawid IB: Expression pattern of the rat Lim-1 homeobox gene suggests a dual role during kidney development. Int J Dev Biol 42: 61-66, 1998.

9. Kobayashi A, Kwan KM, Carroll TJ, McMahon AP, Mendelsohn CL and Behringer RR: Distinct and sequential tissue-specific activities of the LIM-class homeobox gene Lim1 for tubular morphogenesis during kidney development. Development 132: 2809-2823, 2005.

10. Chen YT, Kobayashi A, Kwan KM, Johnson RL and Behringer RR: Gene expression profiles in developing nephrons using Lim1 metanephric mesenchyme-specific conditional mutant mice. BMC Nephrol 7: 1, 2006.

11. Guertl B, Senanayake U, Nusshold E, et al: Lim1, an embryonal transcription factor, is absent in multicystic renal dysplasia, but reactivated in nephroblastomas. Pathobiology 78: 210-219, 2011.

12. Dormoy V, Béraud C, Lindner V, Thomas L, Coquard C, Barthelmebs M, Jacqmin D, Lang H and Massfelder T: LIM-class homeobox gene Lim1, a novel oncogene in human renal cell carcinoma. Oncogene 30: 1753-1763, 2011.

13. Xu Y, Baldassare M, Fisher P, et al: LH-2: a LIM/homeodomain gene expressed in developing lymphocytes and neural cells. Proc Natl Acad Sci USA 90: 227-231, 1993.

14. Richter K, Pinto do O P, Hägglund AC, Wahlin A and Carlsson L: Lhx2 expression in hematopoietic progenitor/stem cells in vivo causes a chronic myeloproliferative disorder and altered globin expression. Haematologica 88: 1336-1347, 2003.

15. Nadal N, Chapiro E, Flandrin-Gresta P, et al: $L H X 2$ deregulation by juxtaposition with the $I G H$ locus in a pediatric case of chronic myeloid leukemia in B-cell lymphoid blast crisis. Leuk Res 36: 195-198, 2012.

16. Wu HK, Heng HH, Siderovski DP, et al: Identification of a human LIM-Hox gene, hLH-2, aberrantly expressed in chronic myelogenous leukaemia and located on 9q33-34.1. Oncogene 12: 1205-1212, 1996.

17. Wu HK and Minden MD: Transcriptional activation of human LIM-HOX gene, $h L H-2$, in chronic myelogenous leukemia is due to a $c i s$-acting effect of $B c r-A b l$. Biochem Biophys Res Commun 233: 806-812, 1997.

18. Gorantla B, Asuthkar S, Rao JS, Patel J and Gondi CS: Suppression of the uPAR-uPA system retards angiogenesis, invasion, and in vivo tumor development in pancreatic cancer cells. Mol Cancer Res 9: 377-389, 2011.

19. Tiede $S$ and Paus R: Lhx2-decisive role in epithelial stem cell maintenance, or just the 'tip of the iceberg'? Bioessays 28: 1157-1160, 2006.

20. Abate-Shen C: Deregulated homeobox gene expression in cancer: cause or consequence? Nat Rev Cancer 2: 777-785, 2002.

21. Rahmatpanah FB, Carstens S, Guo J, et al: Differential DNA methylation patterns of small B-cell lymphoma subclasses with different clinical behavior. Leukemia 20: 1855-1862, 2006.

22. Kim MS, Lee J, Oh T, et al: Genome-wide identification of $O T P$ gene as a novel methylation marker of breast cancer. Oncol Rep 27: 1681-1688, 2012.

23. Kamalakaran S, Varadan V, Giercksky Russnes HE, et al: DNA methylation patterns in luminal breast cancers differ from nonluminal subtypes and can identify relapse risk independent of other clinical variables. Mol Oncol 5: 77-92, 2011.

24. Rauch T, Li H, Wu X and Pfeifer GP: MIRA-assisted microarray analysis, a new technology for the determination of DNA methylation patterns, identifies frequent methylation of homeodomain-containing genes in lung cancer cells. Cancer Res 66: 7939-7947, 2006.

25. Sharma K, Sheng HZ, Lettieri K, et al: LIM homeodomain factors Lhx3 and Lhx4 assign subtype identities for motor neurons. Cell 95: 817-828, 1998. 
26. Sheng HZ, Zhadanov AB, Mosinger B Jr, et al: Specification of pituitary cell lineages by the LIM homeobox gene Lhx3. Science 272: 1004-1007, 1996.

27. Sheng HZ, Moriyama K, Yamashita T, et al: Multistep control of pituitary organogenesis. Science 278: 1809-1812, 1997.

28. Thaler JP, Lee SK, Jurata LW, Gill GN and Pfaff SL: LIM factor Lhx 3 contributes to the specification of motor neuron and interneuron identity through cell-type-specific protein-protein interactions. Cell 110: 237-249, 2002.

29. Dietrich D, Lesche R, Tetzner R, et al: Analysis of DNA methylation of multiple genes in microdissected cells from formalin-fixed and paraffin-embedded tissues. J Histochem Cytochem 57: 477-489, 2009

30. Li H, Witte DP, Branford WW, et al: Gsh-4 encodes a LIM-type homeodomain, is expressed in the developing central nervous system and is required for early postnatal survival. EMBO J 13 2876-2885, 1994.

31. Liu Y, Fan M, Yu S, et al: cDNA cloning, chromosomal localization and expression pattern analysis of human LIM-homeobox gene LHX4. Brain Res 928: 147-155, 2002.

32. Machinis K, Pantel J, Netchine I, et al: Syndromic short stature in patients with a germline mutation in the LIM homeobox LHX4. Am J Hum Genet 69: 961-968, 2001.

33. Raetzman LT, Ward R and Camper SA: Lhx4 and Propl are required for cell survival and expansion of the pituitary primordia. Development 129: 4229-4239, 2002.

34. Kawamata N, Sakajiri S, Sugimoto KJ, Isobe Y, Kobayashi H and Oshimi K: A novel chromosomal translocation $\mathrm{t}(1 ; 14)(\mathrm{q} 25 ; \mathrm{q} 32)$ in pre-B acute lymphoblastic leukemia involves the LIM homeodomain protein gene, Lhx4. Oncogene 21: 4983-4991, 2002.

35. Yamaguchi M, Yamamoto K and Miura O: Aberrant expression of the LHX4 LIM homeobox gene caused by $\mathrm{t}(1 ; 14)(\mathrm{q} 25 ; \mathrm{q} 32)$ in chronic myelogenous leukemia in biphenotypic blast crisis Genes Chromosomes Cancer 38: 269-273, 2003.

36. de Bruijn DR, van Dijk AH, Willemse MP and van Kessel AG The $C$ terminus of the synovial sarcoma-associated SSX proteins interacts with the LIM homeobox protein LHX4. Oncogene 27: 653-662, 2008

37. Goc A, Kochuparambil ST, Al-Husein B, Al-Azayzih A, Mohammad S and Somanath PR: Simultaneous modulation of the intrinsic and extrinsic pathways by simvastatin in mediating prostate cancer cell apoptosis. BMC Cancer 12: 409, 2012.

38. Hung TM, Hu RH, Ho CM, et al: Downregulation of alpha-fetoprotein expression by LHX4: a critical role in hepatocarcinogenesis. Carcinogenesis 32: 1815-1823, 2011.

39. Zhao Y, Hermesz E, Yarolin MC and Westphal H: Genomic structure, chromosomal localization and expression of the human LIM-homeobox gene LHX5. Gene 260: 95-101, 2000.

40. Sheng HZ, Bertuzzi S, Chiang C, et al: Expression of murine Lhx 5 suggests a role in specifying the forebrain. Dev Dyn 208 266-277, 1997.

41. Zhao Y, Sheng HZ, Amini R, et al: Control of hippocampal morphogenesis and neuronal differentiation by the LIM homeobox gene Lhx5. Science 284: 1155-1158, 1999.

42. Grigoriou M, Tucker AS, Sharpe PT and Pachnis V: Expression and regulation of $L h x 6$ and $L h x 7$, a novel subfamily of LIM homeodomain encoding genes, suggests a role in mammalian head development. Development 125: 2063-2074, 1998.

43. Estécio MR, Youssef EM, Rahal P, et al: LHX6 is a sensitive methylation marker in head and neck carcinomas. Oncogene 25 5018-5026, 2006.

44. Jung S, Jeong D, Kim J, et al: Epigenetic regulation of the potential tumor suppressor gene, $h L H X 6.1$, in human cervical cancer. Int J Oncol 38: 859-869, 2011.

45. Jung S, Jeong D, Kim J, et al: The role of $h L H X 6-H M R$ as a methylation biomarker for early diagnosis of cervical cancer. Oncol Rep 23: 1675-1682, 2010.

46. Failli V, Rogard M, Mattei MG, Vernier P and Rétaux S: Lhx 9 and $\operatorname{Lhx} 9 \alpha$ LIM-homeodomain factors: genomic structure expression patterns, chromosomal localization, and phylogenetic analysis. Genomics 64: 307-317, 2000

47. Vladimirova V, Mikeska T, Waha A, et al: Aberrant methylation and reduced expression of LHX9 in malignant gliomas of childhood. Neoplasia 11: 700-711, 2009.

48. Bennett LB, Schnabel JL, Kelchen JM, et al: DNA hypermethylation accompanied by transcriptional repression in follicular lymphoma. Genes Chromosomes Cancer 48: 828-841, 2009.

49. Karlsson O, Thor S, Norberg T, Ohlsson H and Edlund T: Insulin gene enhancer binding protein Isl-1 is a member of a novel class of proteins containing both a homeo-and a Cys His domain. Nature 344: 879-882, 1990
50. Ahlgren U, Pfaff SL, Jessell TM, Edlund T and Edlund $\mathrm{H}$ : Independent requirement for ISL1 in formation of pancreatic mesenchyme and islet cells. Nature 385: 257-260, 1997.

51. Yamada T, Pfaff SL, Edlund T and Jessell TM: Control of cell pattern in the neural tube: motor neuron induction by diffusible factors from notochord and floor plate. Cell 73: 673-686, 1993.

52. Jensen J: Gene regulatory factors in pancreatic development. Dev Dyn 229: 176-200, 2004.

53. Dong J, Asa SL and Drucker DJ: Islet cell and extrapancreatic expression of the LIM domain homeobox gene isl-1. Mol Endocrinol 5: 1633-1641, 1991.

54. Graham RP, Shrestha B, Caron BL, et al: Islet-1 is a sensitive but not entirely specific marker for pancreatic neuroendocrine neoplasms and their metastases. Am J Surg Pathol 37: 399-405, 2013.

55. Koo J, Mertens RB, Mirocha JM, Wang HL and Dhall D: Value of Islet 1 and PAX8 in identifying metastatic neuroendocrine neoplasms of pancreatic origin. Mod Pathol 25: 893-902, 2012.

56. Schmitt AM, Riniker F, Anlauf M, et al: Islet 1 (Isl1) expression is a reliable marker for pancreatic endocrine neoplasms and their metastases. Am J Surg Pathol 32: 420-425, 2008.

57. Hermann G, Konukiewitz B, Schmitt A, Perren A and Klöppel G: Hormonally defined pancreatic and duodenal neuroendocrine tumors differ in their transcription factor signatures: expression of ISL1, PDX1, NGN3, and CDX2. Virchows Arch 459: 147-154, 2011.

58. Cheung IY, Feng Y, Gerald W and Cheung NK: Exploiting gene expression profiling to identify novel minimal residual disease markers of neuroblastoma. Clin Cancer Res 14: 7020-7027, 2008.

59. Chizhikov VV, Lindgren AG, Mishima Y, et al: Lmxla regulates fates and location of cells originating from the cerebellar rhombic lip and telencephalic cortical hem. Proc Natl Acad Sci USA 107: 10725-10730, 2010.

60. Cai J, Donaldson A, Yang M, German MS, Enikolopov G and Iacovitti L: The role of Lmxla in the differentiation of human embryonic stem cells into midbrain dopamine neurons in culture and after transplantation into a Parkinson's disease model. Stem Cell 27: 220-229, 2009.

61. Lin CK, Chao TK, Lai HC and Lee HS: LMX1A as a prognostic marker in ovarian mucinous cystadenocarcinoma. Am J Clin Pathol 137: 971-977, 2012

62. Tsai WC, Lee HS, Lin CK, Chen A, Nieh S and Ma HI: The association of osteopontin and LMX1A expression with World Health Organization grade in meningiomas and gliomas. Histopathology 61: 844-856, 2012.

63. Tsai WC, Lin CK, Yang YS, et al: The correlations of LMX1A and osteopontin expression to the clinicopathologic stages in pancreatic adenocarcinoma. Appl Immunohistochem Mol Morphol 21: 395-400, 2013.

64. Liu CY, Chao TK, Su PH, et al: Characterization of LMX-1A as a metastasis suppressor in cervical cancer. J Pathol 219: 222-231, 2009.

65. Lai HC, Lin YW, Huang TH, et al: Identification of novel DNA methylation markers in cervical cancer. Int J Cancer 123: 161-167, 2008.

66. Lai HC, Lin YW, Huang RL, et al: Quantitative DNA methylation analysis detects cervical intraepithelial neoplasms type 3 and worse. Cancer 116: 4266-4274, 2010.

67. Chao TK, Yo YT, Liao YP, et al: LIM-homeobox transcription factor 1, alpha (LMX1A) inhibits tumourigenesis, epithelialmesenchymal transition and stem-like properties of epithelial ovarian cancer. Gynecol Oncol 128: 475-482, 2013.

68. Su HY, Lai HC, Lin YW, et al: An epigenetic marker panel for screening and prognostic prediction of ovarian cancer. Int $\mathrm{J}$ Cancer 124: 387-393, 2009.

69. Zhao Y, Guo S, Sun J, et al: Methylcap-seq reveals novel DNA methylation markers for the diagnosis and recurrence prediction of bladder cancer in a Chinese population. PLoS One 7: e35175, 2012.

70. Dong W, Feng L, Xie Y, Zhang H and Wu Y: Hypermethylationmediated reduction of LMX1A expression in gastric cancer. Cancer Sci 102: 361-366, 2011

71. Paz MF, Wei S, Cigudosa JC, et al: Genetic unmasking of epigenetically silenced tumor suppressor genes in colon cancer cells deficient in DNA methyltransferases. Hum Mol Genet 12: 2209-2219, 2003.

72. Chen H, Lun Y, Ovchinnikov D, et al: Limb and kidney defects in $L m x 1 b$ mutant mice suggest an involvement of $L M X 1 B$ in human nail patella syndrome. Nat Genet 19: 51-55, 1998. 
73. Riddle RD, Ensini M, Nelson C, Tsuchida T, Jessell TM and Tabin C: Induction of the LIM homeobox gene Lmxl by WNT7a establishes dorsoventral pattern in the vertebrate limb. Cell 83: 631-640, 1995

74. Rieger ME, Sims AH, Coats ER, Clarke RB and Briegel KJ: The embryonic transcription cofactor $\mathrm{LBH}$ is a direct target of the Wnt signaling pathway in epithelial development and in aggressive basal subtype breast cancers. Mol Cell Biol 30: 4267-4279, 2010.

75. Kengaku M, Capdevila J, Rodriguez-Esteban C, et al: Distinct WNT pathways regulating AER formation and dorsoventral polarity in the chick limb bud. Science 280: 1274-1277, 1998.

76. Clevers $\mathrm{H}$ : Wnt/ $\beta$-catenin signaling in development and disease. Cell 127: 469-480, 2006.

77. Millen KJ, Millonig JH and Hatten ME: Roof plate and dorsal spinal cord dl1 interneuron development in the dreher mutant mouse. Dev Biol 270: 382-392, 2004.

78. Chizhikov VV and Millen KJ: Control of roof plate formation by Lmx1a in the developing spinal cord. Development 131: 2693-2705, 2004.

79. Kishigami S and Mishina Y: BMP signaling and early embryonic patterning. Cytokine Growth Factor Rev 16: 265-278, 2005.

80. Dormoy V, Jacqmin D, Lang H and Massfelder T: From development to cancer: lessons from the kidney to uncover new therapeutic targets. Anticancer Res 32: 3609-3617, 2012.

81. Sato A and Shibuya H: WNK signaling is involved in neural development via Lhx8/Awh expression. PLoS One 8: e55301, 2013.

82. Moriguchi T, Urushiyama S, Hisamoto N, et al: WNK1 regulates phosphorylation of cation-chloride-coupled cotransporters via the STE20-related kinases, SPAK and OSR1. J Biol Chem 280 42685-42693, 2005.

83. Vitari AC, Deak M, Morrice NA and Alessi DR: The WNK1 and WNK4 protein kinases that are mutated in Gordon's hypertension syndrome phosphorylate and activate SPAK and OSR 1 protein kinases. Biochem J 391: 17-24, 2005.
84. Moniz S and Jordan P: Emerging roles for WNK kinases in cancer. Cell Mol Life Sci 67: 1265-1276, 2010.

85. Veríssimo $\mathrm{F}$ and Jordan P: WNK kinases, a novel protein kinase subfamily in multi-cellular organisms. Oncogene 20: 5562-5569, 2001.

86. Tu SW, Bugde A, Luby-Phelps K and Cobb MH: WNK1 is required for mitosis and abscission. Proc Natl Acad Sci USA 108: 1385-1390, 2011.

87. Sun X, Gao L, Yu RK and Zeng G: Down-regulation of WNK1 protein kinase in neural progenitor cells suppresses cell proliferation and migration. J Neurochem 99: 1114-1121, 2006.

88. Moniz S and Jordan P: Emerging roles for WNK kinases in cancer. Cell Mol Life Sci 67: 1265-1276, 2010.

89. Cohen ED, Wang Z, Lepore JJ, et al: Wnt/ $\beta$-catenin signaling promotes expansion of Isl-1-positive cardiac progenitor cells through regulation of FGF signaling. J Clin Invest 117: 1794-1804, 2007.

90. Liem KF Jr, Jessell TM and Briscoe J: Regulation of the neural patterning activity of sonic hedgehog by secreted BMP inhibitors expressed by notochord and somites. Development 127 : 4855-4866, 2000.

91. Chizhikov VV and Millen KJ: Control of roof plate development and signaling by Lmxlb in the caudal vertebrate CNS. J Neurosci 24: 5694-5703, 2004.

92. Chizhikov VV and Millen KJ: Control of roof plate formation by Lmx1a in the developing spinal cord. Development 131: 2693-2705, 2004.

93. Alexandre P, Bachy I, Marcou M and Wassef M: Positive and negative regulations by FGF8 contribute to midbrain roof plate developmental plasticity. Development 133: 2905-2913, 2006. 